\title{
ASYMPTOTICS FOR THE TIME-DEPENDENT THERMISTOR PROBLEM
}

\author{
BY
}

\author{
GIOVANNI CIMATTI
}

Department of Mathematics, via Buonarroti, 2, 56100 Pisa, Italy

\begin{abstract}
Using the Liapunoff method, the global asymptotic stability of the stationary solution of the time-dependent thermistor problem is proved.

In this paper we study a special case of the time-dependent thermistor problem, for which it is possible to give a fairly complete description of the asymptotic behaviour of the solution. A difference of potential $V$, in series with an ordinary resistor $R$, is applied to an indefinite slab of width $d$ and electric conductivity $\sigma(u)$. We model this situation with the following one-dimensional initial-boundary value problem $(D)$ :

$$
\begin{aligned}
\left(\sigma(u) \varphi_{x}\right)_{x} & =0 \\
\varphi(0, t) & =0 \\
V-R \sigma(0) \varphi_{x}(d, t)-\varphi(d, t) & =0 \\
u_{t}-u_{x x} & =\sigma(u) \varphi_{x}^{2} \\
u(0, t)=u(d, t) & =0 \\
u(x, 0) & =u_{0}(x)
\end{aligned}
$$

where $\varphi(x, t)$ is the electric potential and $u(x, t)$ the temperature.

In the usual thermistor problem, $R$ is taken equal to 0 , but this ignores e.g. the internal resistance of the generator. Thus, the present model is physically more realistic. The method is based on the use of a Liapunoff function. We treat in details the case

$$
\sigma(u)=\sigma_{0} e^{u},
$$

which is relevant in the theory of $P T C$ thermistor [5].

In recent years, the thermistor problem has been the subject of many mathematical investigations. We quote, among others, the work of W. Allegretto, W. Lin ([1], [2]), and A. Lacey ([8], [9]) for the time-dependent case and, for the steady case, the papers of S. Howison, F. Rodrigues, and M. Shilor ([6], [7]).

Received December 19, 2002.

2000 Mathematics Subject Classification. Primary 78A97, 34C25.

E-mail address: cimatti@dm.unipi.it 
We consider first the stationary problem $(S)$ corresponding to $(D)$, i.e.:

$$
\begin{gathered}
\left(\sigma(u) \varphi^{\prime}\right)^{\prime}=0 \\
\varphi(0)=0 \\
V-R \sigma(0) \varphi^{\prime}(d)-\varphi(d)=0 \\
-u^{\prime \prime}=\sigma(u) \varphi^{\prime 2} \\
u(0)=u(d)=0 .
\end{gathered}
$$

When $R=0$, problem $(S)$ has one and only one solution if $\ell=\int_{0}^{\infty} \frac{d t}{\sigma(t)}>\frac{V^{2}}{8}$ and no solution when $\frac{V^{2}}{8} \geq \ell$. We refer for more details to [6]. In the present situation there is always one and only one solution. This can easily be understood on physical grounds, since the electric current cannot exceed a fixed value determined by the presence of $R$. A formal proof is the following. Let $F:[0, \infty) \rightarrow[0, \ell)$

$$
F(u)=\int_{0}^{u} \frac{d t}{\sigma(t)}
$$

and

$$
\theta=\frac{\varphi^{2}}{2}+F(u), \Gamma=\varphi(d) .
$$

If $(u(x), \varphi(x))$ is a solution to problem $(S)$ we have

$$
\begin{aligned}
\left(\sigma(u) \theta^{\prime}\right)^{\prime} & =0 \\
\theta(0)=0, \theta(d) & =\frac{\Gamma^{2}}{2} \\
\left(\sigma(u) \varphi^{\prime}\right)^{\prime} & =0 \\
\varphi(0)=0, \varphi(d) & =\Gamma .
\end{aligned}
$$

Whence it follows the functional relation

$$
\theta(x)=\frac{\Gamma \varphi(x)}{2}
$$

Let $u=G(w), G:[0, \ell) \rightarrow[0, \infty)$ be the inverse function of $w=F(u)$. From (14) and (19) we have

$$
u(x)=G\left(\frac{\Gamma \varphi(x)}{2}-\frac{\varphi^{2}(x)}{2}\right) .
$$

Define the bijection $L:[0, \Gamma] \rightarrow[0, L(\Gamma)], \psi=L(\varphi)$ via

$$
L(\varphi)=\int_{0}^{\varphi} \sigma\left(G\left(\frac{t \Gamma}{2}-\frac{t^{2}}{2}\right)\right) d t
$$

and $\psi(x)=L(\varphi(x))$. By (20) and (17) we have $\psi^{\prime \prime}(x)=0, \psi(0)=0$, and $\psi(d)=$ $L(\varphi(d))=L(\Gamma)$. Hence $\psi(x)=\frac{x L(\Gamma)}{d}$ and $\psi^{\prime}(d)=\frac{L(\Gamma)}{d}$. Thus condition (10) becomes

$$
V-\Gamma=\frac{R}{d} L(\Gamma)
$$

where

$$
L(\Gamma)=\int_{0}^{\Gamma} \sigma\left(G\left(\frac{t \Gamma}{2}-\frac{t^{2}}{2}\right)\right) d t
$$


and, by direct computation, we find

$$
L(\Gamma)=\frac{8}{\sqrt{8-\Gamma^{2}}} \arctan \left[\frac{\Gamma}{\sqrt{8-\Gamma^{2}}}\right] .
$$

The solutions of problem $(S)$ are in a one-to-one correspondence with those of Eq. (21) and, by clementary means, it can be seen that $(21)$ has a unique solution $\tilde{\Gamma}$. Hence the only solution to problem $(S)$ is given by:

$$
\tilde{\varphi}(x)=L^{-1}\left(\frac{x L(\tilde{\Gamma})}{d}\right), \tilde{u}(x)=G\left(\frac{\tilde{\Gamma} \tilde{\varphi}(x)}{2}-\frac{\tilde{\varphi}^{2}(x)}{2}\right) .
$$

The norm in $L^{2}(0, d)$ is denoted by \|\| . Other norms are identified by subscript.

TheOrem 1. Let $T>0, u_{0}(x) \in H_{0}^{1}(0, d)$, and $u_{0 m}=\min u_{0}(x)$. Then, there exists one and only one solution to problem $(D)$ such that

$$
u(x, t) \in L^{2}\left(0, T ; H^{2}(0, d)\right) \cap L^{\infty}\left(0, T ; H_{0}^{1}(0, d)\right), u_{t}(x, t) \in L^{2}\left(0, T ; L^{2}(0, d)\right) .
$$

Moreover,

$$
\operatorname{ess} \sup _{[0, T]}\|u(t)\|_{H_{0}^{1}(0, d)}+\|u\|_{L^{2}\left(0, T ; H^{2}(0, d)\right)}+\left\|u_{t}\right\|_{L^{2}\left(0, T ; L^{2}(0, d)\right)} \leq C\left\|u_{0}\right\|_{H_{0}^{1}(0, d)}
$$

and

$$
\begin{aligned}
& 0 \leq \varphi(x, t) \leq V \\
& 0 \leq u(x, t) \leq U_{M},
\end{aligned}
$$

where $U_{M I}=\max U(x, t)$ and $U(x, t)$ is solution of the linear problem:

$$
\begin{aligned}
U_{t}-U_{x x} & =\frac{V^{2}}{R \sigma\left(u_{0 m}\right)} \\
U(0, t) & =U(d, t)=0 \\
U(x, 0) & =u_{0}(x) .
\end{aligned}
$$

Proof. Let $(\varphi, u)$ be a solution of problem $(D)$. Multiplying $(1)$ by $\varphi(x, t)$ we have, integrating by parts,

$$
\varphi(d, t) \sigma(0) \varphi_{x}(d, t)=\int_{0}^{d} \sigma(u(x, t)) \varphi_{x}^{2}(x, t) d x
$$

which implies (24) recalling (3). In view of the positivity of the right hand side of (4), we have $u(x, t) \geq u_{0 m}$. Moreover, integrating (1) with respect to $x$ and taking into account of $(24)$, we obtain the "a priori" estimate

$$
\sigma(u(x, t)) \varphi_{x}^{2}(x, t)=\frac{(V-\varphi(d, t))^{2}}{R^{2} \sigma(u(d, t))} \leq \frac{V^{2}}{R^{2} \sigma\left(u_{0 m}\right)} .
$$

Therefore the parabolic maximum principle implies (25). Let $\tilde{\sigma}(\xi) \in C^{\infty}(\mathbf{R})$ satisfy: (i) $\tilde{\sigma}(\xi)=\sigma_{0} e^{\xi}$, if $-\infty<\xi \leq U_{m}$; (ii) $\tilde{\sigma}(\xi)=\sigma_{0} e^{U_{m}}$, if $\xi \geq 2 U_{M}$; (iii) $\tilde{\sigma}^{\prime}(\xi) \geq 0$. The 
method of Galerkin can be used in view of the boundedness of $\tilde{\sigma}$ to prove the existence of a solution of the following auxiliary problem $(A)$ :

$$
\begin{gathered}
\left(\tilde{\sigma}(u) \varphi_{x}\right)_{x}=0 \\
\varphi(0, t)=0, V-R \tilde{\sigma}(0) \varphi_{x}(d, t)-\varphi(d, t)=0 \\
u_{t}-u_{x x}=\tilde{\sigma}(u) \varphi_{x}^{2}=0 \\
u(0, t)=u(d, t)=0 \\
u(x, 0)=u_{0}(x) .
\end{gathered}
$$

On the other hand, the "a priori" estimate (25) holds for the solutions of $(A)$ and this guarantees the existence of a solution of $(D)$. Uniqueness can be obtained reasoning as in [3] with application of the Gronwall inequality.

The solution of problem $(D)$ defines a dynamical system

$$
S(t): H_{0}^{1}(0, d) \rightarrow H_{0}^{1}(0, d), t \geq 0, u(t)=S(t) u_{0},
$$

since $u \in C\left([0, \infty) ; H_{0}^{1}(0, d)\right)$. The solution of the stationary problem $(S)$ gives the only fixed point $\tilde{u}$ of $S(t)$. In the next theorem we prove that $\tilde{u}$ is globally asymptotically stable in $H_{0}^{1}(0, d)$.

Theorem 2. Let $(u(x, t), \varphi(x, t))$ and $(\tilde{u}(x), \tilde{\varphi}(x))$ be solutions to problem $(D)$ and $(S)$ respectively. Then

$$
\begin{aligned}
& \lim _{t \rightarrow \infty}\|u(t)-\tilde{u}\|_{H_{0}^{1}(0, d)}=0 \\
& \lim _{t \rightarrow \infty}\|\varphi(t)-\tilde{\varphi}\|_{H_{0}^{1}(0, d)}=0 .
\end{aligned}
$$

Proof. Let $\Phi(u): H_{0}^{1}(0, d) \rightarrow \mathbf{R}$ be the continuous functional

$$
\Phi(u)=\int_{0}^{d}\left(\frac{1}{2} u^{\prime 2}-\sigma(u) \varphi^{\prime 2}\right) d x-\frac{1}{R}[\varphi(d)-V]^{2},
$$

where $\varphi=T(u)$ is solution to problem

$$
\begin{aligned}
\left(\sigma(u) \varphi^{\prime}\right)^{\prime} & =0 \\
\varphi(0) & =0, V-R \sigma(0) \varphi^{\prime}(d)-\varphi(d)=0 .
\end{aligned}
$$

By (24) and (29), $\Phi(u)$ is bounded below on the solutions of problem $(D)$. Using the choice $\sigma(u)=\sigma_{0} e^{u}$ and integrating by parts we find, on the solutions of problem $(D)$,

$$
\frac{d}{d t} \Phi(u(t))=-\int_{0}^{d}\left(u_{x x}+\sigma(u) \varphi_{x}^{2}\right)^{2} d x \leq 0 .
$$

Recalling (23) we conclude that every orbit $u\left(t ; u_{0}\right)$ is bounded in $H_{0}^{1}(0, d)$. We claim that $u\left(t ; u_{0}\right)$ is also compact in $H_{0}^{1}(0, d)$ if $t \geq t_{0}>0$. Indeed, let $A=\frac{d^{2}}{d x^{2}}$ and $D(A)=$ $H_{0}^{1}(0, d) \cap H^{2}(0, d)$. The operator $A$ is positive, selfadjoint, and with a compact resolvent. Thus, we can define $A^{\alpha}$ and the corresponding Banach spaces $D\left(A^{\alpha}\right)$ equipped with the graph norm $\|u\|_{\alpha}=\left\|A^{\alpha} u\right\|$. In particular $D\left(A^{1 / 2}\right)=H_{0}^{1}(0, d)$. Moreover, the following estimate holds:

$$
\left\|A^{\alpha} e^{A t} u\right\| \leq C t^{-\alpha}\|u\|
$$


If $0<\beta<\alpha$, we have the compact inclusion $D\left(A^{\alpha}\right) \subset D\left(A^{\beta}\right)$. Let $u \in H_{0}^{1}(0, d)$ and $f(u)=\tilde{\sigma}(u)\left(T(u)^{\prime}\right)^{2}=\tilde{\sigma}(u) \varphi^{\prime 2}$. With these notations, problem $(D)$ can be written as an abstract equation of evolution

$$
u^{\prime}=A u+f(u), u(0)=u_{0} .
$$

Using the variation of constant formula we have

$$
u\left(t ; u_{0}\right)=e^{A t} u_{0}+\int_{0}^{t} e^{A(t-s)} f(u(s)) d s .
$$

Noting that $f$ maps bounded subsets of $H_{0}^{1}(0 . d)$ into bounded subsets of $L^{2}(0, d)$, we have, for all $t \geq 0$,

$$
\left\|f\left(u\left(t ; u_{0}\right)\right)\right\| \leq M
$$

since orbits $u\left(t ; u_{0}\right)=S(t) u_{0}$ are bounded in $H_{0}^{1}(0, d)$. Let $\frac{1}{2}<\beta<1$. From (35) and (36) we have, by (33),

$$
\begin{aligned}
\left\|u\left(t ; u_{0}\right)\right\|_{\beta} \leq\left\|e^{A t} u_{0}\right\|_{\beta} & +\int_{0}^{t}\left\|e^{A(t-s)} f(u(s))\right\|_{\beta} d s=\left\|A^{\beta} e^{A t} u_{0}\right\| \\
& +\int_{0}^{t}\left\|A^{\beta} e^{A(t-s)} f(u(s))\right\| d s \\
\leq C t^{-\beta}\left\|u_{0}\right\| & +\int_{0}^{t} C(t-s)^{-\beta} M d s=C t^{-\beta}\left\|u_{0}\right\|+\frac{C M t^{1-\beta}}{1-\beta} .
\end{aligned}
$$

If $t \geq t_{0}>0$, the orbit $u\left(t ; u_{0}\right)$ is bounded in $D\left(A^{\beta}\right)$ and thus compact in $H_{0}^{1}(0, d)$. $\Phi\left(S(t) u_{0}\right)$ is nonincreasing; hence $\ell=\lim _{t \rightarrow \infty} \Phi\left(S(t) u_{0}\right)$ exists and, by compactness, a subsequence $t_{k} \rightarrow \infty$ can be found such that

$$
\left\|u\left(t_{k} ; u_{0}\right)-\tilde{u}\right\|_{H_{0}^{1}(0, d)} \text {. as } k \rightarrow \infty .
$$

Therefore $\Phi(\tilde{u})=\ell$. On the other hand, $\ell \leq \Phi(S(t) \bar{u}) \leq \Phi(\tilde{u})=\ell$ and $\frac{d}{d t} \Phi(S(t) \tilde{u})=0$. So, by $(32)$, we conclude that $(\tilde{\varphi}=T(\tilde{u}), \tilde{u})$ is a solution to the stationary problem $(S)$. This solution, however, is unique; thus the entire sequence converges and we obtain $(30)$. By difference from (1) and (8), multiplying the result by $\tilde{\varphi}-\varphi$ and integrating by parts, we have

$$
\frac{1}{R}[\tilde{\varphi}(\ell)-\varphi(d, t)]^{2}+\int_{0}^{d} \sigma(\tilde{u})\left(\varphi^{\prime}-\varphi_{x}\right)^{2} d x=-\int_{0}^{d} \varphi_{x}[\sigma(\tilde{u})-\sigma(u)]\left(\tilde{\varphi}^{\prime}-\varphi_{x}\right) d x .
$$

This implies (31).

\section{REFERENCES}

[1] Allegretto, W., Lin, Y., and Ma, S., Existence and long time behaviour of solutions to obstacle thermistor equations, Discrete and Continuous Dynamical Systems, 8 (2002), 757-780.

[2] Allegretto, W., and Xie, H., A non-local thermistor problem, European J. of Appl. Math., 6, (1995), 83-94.

[3] Antontsev, S. N., and Chipot, M., The thermistor problem: existence, smoothness, uniqueness and blow up, SIAM J. Math. Anal., 25 (1994), 209-223.

[4] Cimatti, G., On the stability of the solution of the thermistor problem, Appl. Anal., 73 (1999), 407-423.

[5] Hyde, F. J., Thermistors, Iliffe Books (1971), London.

[6] Howison, S. D., A note on the thermistor problem in two space dimensions, Quart. Appl. Math., $98(1989), 37-39$. 
[7] Howison, S. D., Rodrigues. J. F., and Shilor, M., Stationary solutions to the thermistor problem, J. Math. Anal. Appl. 174 (1993), 573-588.

[8] Lacey, A., Thermal runaway in a nonlocal problem modelling Ohmic heating: Part I: Model derivation and some special cases, Euro Jnl. Appl. Math, 6 (1995), 127-144.

[9] Lacey, A., Thermal runaway in a nonlocal problem modelling Ohmic heating: Part II: General proof of blow-up and asymptotic of runaway, Euro Jnl. Appl. Math, 6 (1995), 201-224. 